\title{
Multifraction radiotherapy for palliation of painful bone metastases: 20 Gy versus $30 \mathrm{~Gy}$
}

\author{
Maurizio Valeriani ${ }^{1}$, Claudia Scaringi ${ }^{1}$, Luciana Blasi ${ }^{2}$, Alessia Carnevale ${ }^{1}$, Vitaliana De Sanctis ${ }^{1}$, Paolo Bonome ${ }^{1}$, \\ Stefano Bracci ${ }^{1}$, Gianluca Marrone ${ }^{1}$, Giuseppe Minniti ${ }^{1}$, Riccardo Maurizi Enrici ${ }^{1}$ \\ ${ }^{1}$ Department of Radiation Oncology, Sant'Andrea Hospital, University "Sapienza", Rome - Italy \\ ${ }^{2}$ Department of Anaesthesiology and Intensive Care, Sant'Andrea Hospital, University "Sapienza", Rome - Italy
}

\begin{abstract}
Aims and Background: To compare 2 multifraction radiotherapy schedules in the palliation of painful bone metastases.

Methods and Study design: We retrospectively analyzed clinical data of 105 patients with a total of 140 painful bone metastases who were treated with $20 \mathrm{~Gy}$ in 5 fractions or $30 \mathrm{~Gy}$ in 10 fractions. The primary tumors were breast $(30 \%)$, lung $(28 \%)$, and prostate $(14 \%)$. The main sites of irradiation were spine $(n=79)$ and sacrum or pelvis $(n=39)$. Pain was graded by patients according to the pain numeric rating scale just before and 1 month after radiotherapy. Pain progression was defined as an increase $\geq 2$ on pain scale after an initial response.

Results: The overall response rate at 1 month was $88.6 \%$. Overall response rate was $89.6 \%$ in the $20-G y$ arm and $87.3 \%$ in the $30-G y$ arm $(p=0.669)$. The rate of complete response was statistically better in patients treated with $30 \mathrm{~Gy}(\mathrm{p}=0.019)$. The mean reduction in pain was 3.2 in the $20-\mathrm{Gy}$ group and 3.6 in the $30-\mathrm{Gy}$ group. Pain progression was $6.5 \%$ and $1.6 \%$, respectively. The incidence of acute toxicity was statistically significantly higher in the $30-G y \operatorname{arm}(23.8 \%)$ than in the $20-G y$ arm $(2.6 \%)(p=0.001)$. One pathologic fracture of the irradiated bone was observed in the 30-Gy arm. Two lesions, one in each group, were re-irradiated for pain recurrence. Pain progression was found in $6.5 \%$ of the irradiated lesions in the $20-\mathrm{Gy}$ arm and in $1.6 \%$ in the $30-\mathrm{Gy}$ arm.

Conclusions: In our series, both regimens achieved high rate of pain relief, although the group treated with higher total dose reported better complete response rate. The 30-Gy arm had a significantly higher rate of acute toxicity.
\end{abstract}

Keywords: Bone metastases, Multifraction radiotherapy, Palliation

\section{Introduction}

Bone metastases are one of the most common manifestations of metastatic disease in patients with cancer and are the third most common site of distant metastases after liver and lung (1). Autopsy series report evidence of bone metastases in up to $85 \%$ of patients with cancer, depending on the primary tumor (2). The incidence and prognosis of bone metastases varies with primary sites. Metastases to the bone occur in up to $70 \%$ of prostate and breast cancer cases and in up to $40 \%$ of lung, renal cell carcinoma, and thyroid cancer cases (3).

On the basis of associated symptoms, bone metastases are classified as complicated and uncomplicated. The main

Accepted: January 30, 2015

Published online:

Corresponding author:

Claudia Scaringi

Sant'Andrea Hospital

Department of Radiation Oncology

Via di Grottarossa 1035

00189 Rome, Italy

clascaringi@gmail.com symptom of uncomplicated bone metastases is severe pain, which causes a substantial burden in morbidity and contributes to the decreased quality of life experienced by these patients. In case of uncomplicated bone metastases, the aim of treatment is palliation of pain. Control of symptoms can be achieved with the use of radiotherapy (RT) and analgesic drugs, while bisphosphonates can be administered to prevent and reduce the incidence of skeletal-related events. Improvement of pain with RT is obtained in $50 \%-80 \%$ of patients and complete pain relief is obtained in $20 \%-50 \%$ of patients (4, $5)$. In the presence of pathologic fractures and spinal cord compression, bone metastases are defined as complicated (6). Treatment of pathologic fracture consists of surgical stabilization, with adjuvant RT to improve recalcification of the osteolytic bone. Spinal cord compression represents a complication that requires urgent treatment to avoid irreversible neurologic deficits. The management of spinal cord compression includes decompressive surgery or RT delivered in single or multiple fractions (4).

Different RT schedules are used for uncomplicated bone metastases treatment: $40 \mathrm{~Gy}$ in 20 fractions, $30 \mathrm{~Gy}$ in 10 fractions, $20 \mathrm{~Gy}$ in 5 fractions, and single fractions of $10 \mathrm{~Gy}$, $8 \mathrm{~Gy}, 6 \mathrm{~Gy}$, or $4 \mathrm{~Gy}$ (7-9). The most appropriate fractionation schedule is a matter of debate, but several randomized trials 
have compared short versus long course treatments, finding no difference in terms of pain relief (10-13). However, it was observed that patients receiving a single fraction of RT experienced recurrence of pain and required re-irradiation to the same site more often compared to patients receiving multiple fractions (14).

In this report, we review our experience in patients with painful uncomplicated bone metastases treated with $20 \mathrm{~Gy}$ in 5 fractions or $30 \mathrm{~Gy}$ in 10 fractions with the aim to compare the palliative effect of these 2 types of fractionation.

\section{Patients and Methods}

Between February 2010 and January 2014, 105 patients with a total of 140 painful bone metastases from different solid tumors were treated with 2 different RT schedules at our institution. Of the 140 lesions, 77 were treated with a radiation dose of 20 Gy delivered in 5 fractions over 1 week and 63 received a radiation dose of $30 \mathrm{~Gy}$ delivered in 10 fractions over 2 weeks.

Patients and tumor characteristics are shown in Table I. There were 59 men and 46 women. Sixty-eight (65\%) patients were treated for a single bone metastasis and 37 (35\%) patients for 2-4 metastases. The median patient age was 64 years (range 32-86). The primary tumors included breast carcinoma (30\%), lung carcinoma (28\%), prostate adenocarcinoma (14\%), and other histologies (28\%). The most common site of bone metastases was the spine $(n=79)$ followed by sacrum or pelvis $(n=39)$ and other sites $(n=22)$. Ninety-three (88\%) patients were taking analgesic drugs at the time of RT. Patients received 3D conformal RT delivered with 6/15 megavoltage photons from a linear accelerator. Target volumes were identified on planning computed tomography images and simulation of treatment fields was performed before treatment. All patients completed the planned RT schedule.

The primary objective of our study was to compare the effectiveness in pain relief of the 2 different RT schedules. The secondary endpoint was to compare acute toxicity, pathologic fracture, spinal cord compression, and retreatment rates between the 2 groups. Pain was graded according to the pain numeric rating scale (NRS) (15). The NRS is a single 11-point numeric scale that assesses pain severity, ranging from 0 to 10 , with 0 indicating absence of pain and 10 indicating maximum pain intensity. The severity of pain was assessed prior to and 1 month after the end of RT. The clinical response to irradiation was classified as complete response (CR), resolution of pain; partial response (PR), improvement $\geq 2$ on the NRS; and no response, no change in pain symptoms or deterioration. Pain progression was defined as an increase in pain $\geq 2$ after an initial response. Toxicity was measured by use of Radiation Therapy Oncology Group criteria (16).

Chi-square test and logistic regression model were used to identify factors that influence the pain response. Pain relief was stratified by $\mathrm{RT}$ schedule, site of treatment, age ( $\leq 50$ years or $>50$ years), sex, histology, and use of analgesic drugs. Limit for statistical significance was $p<0.05$. Chisquare test was used to compare the acute toxicity with dose. Statistical evaluation was performed using Statistical Package for the Social Sciences version 20 (SPSS Inc., SAS, Chicago, IL).
TABLE I - Patients and tumor characteristics

\begin{tabular}{lcc}
\hline & 20 Gy, $\mathbf{n}$ (\%) & $\mathbf{3 0 ~ G y , ~} \mathbf{n}$ (\%) \\
\hline Number of patients & 58 & 47 \\
Number of lesions & 77 & 63 \\
F/M & $28 / 30$ & $25 / 22$ \\
Age, y, median (range) & $68(35-86)$ & $65(32-82)$ \\
Histology & & \\
$\quad$ Breast carcinoma & $18(31)$ & $14(30)$ \\
$\quad$ Lung cancer & $20(35)$ & $9(19)$ \\
$\quad$ Prostate adenocarcinoma & $6(10)$ & $9(19)$ \\
$\quad$ Others & $14(24)$ & $15(32)$ \\
Number of metastases per patient & & \\
$\quad$ Single & $45(77)$ & $31(66)$ \\
$\quad$ Multiple (2-4) & $13(23)$ & $16(34)$ \\
Site of metastases & & \\
$\quad$ Spine & $47(61)$ & $32(51)$ \\
$\quad$ Sacrum or pelvis & $19(25)$ & $20(32)$ \\
$\quad$ Others & $11(14)$ & $11(17)$ \\
\hline
\end{tabular}

TABLE II - Outcomes of 140 irradiated lesions

\begin{tabular}{lcc}
\hline & 20 Gy, \% & 30 Gy, \% \\
\hline Overall response & 89.6 & 87.3 \\
Complete response & 6.5 & 19 \\
Partial response & 83.1 & 68.3 \\
No response & 10 & 13 \\
Pain progression & 6.5 & 1.6 \\
Acute toxicity & 2.6 & 23.8 \\
\hline
\end{tabular}

\section{Results}

Outcomes of treatment are shown in Table II. The overall response rate at 1 month was $88.6 \%$. The overall response rate was $89.6 \%$ (CR and PR rates $6.5 \%$ and $83.1 \%$, respectively) in the $20-$ Gy arm compared with $87.3 \%$ (CR and PR rates $19 \%$ and $68.3 \%$, respectively) in the $30-\mathrm{Gy}$ arm $(p=0.669)$. The rate of $C R$ was statistically better in patients treated with $30 \mathrm{~Gy}(p=0.019)$. The mean reduction in pain was 3.2 in the $20-G y$ group and 3.6 in the $30-G y$ group. Pain progression was found in $6.5 \%$ of the irradiated lesions in the $20-\mathrm{Gy}$ arm and in $1.6 \%$ in the $30-\mathrm{Gy}$ arm. This difference was not statistically significant.

The incidence of acute toxicity was statistically significantly higher in the 30-Gy arm (23.8\%) than in the 20-Gy arm $(2.6 \%)(p=0.001)$. Adverse events were mainly represented by gastrointestinal toxicity, with nausea, vomiting, and diarrhea being the most common acute side effects experienced by patients. Only one patient, in the 30-Gy arm, had grade 4 acute toxicity (diarrhea). Two patients, in the 30-Gy arm, reported mild dysphagia. There was no grade 3-4 toxicity in the 20-Gy group, while grade 1-2 toxicities were represented 
by nausea and vomiting. One patient in the 30-Gy arm experienced pathologic fracture of the irradiated bone 4 months after treatment. Two lesions, one in each group, were reirradiated for pain recurrence 5 and 18 months after initial treatment, respectively.

By logistic regression, no difference in response rate at 1 month was found between the 2 treatment arms when stratified by RT schedule, site of treatment, age ( $\leq 50$ years or $>50$ years), sex, histology, and use of analgesic drugs.

\section{Discussion}

The optimal doses and fractionation for the treatment of symptomatic bone metastases are not well-defined and several randomized trials have compared different schedules concluding that single-fraction $\mathrm{RT}$ is as effective for pain relief as multifraction regimens such as $20 \mathrm{~Gy}$ in 5 fractions or $30 \mathrm{~Gy}$ in 10 fractions, although re-irradiation for recurrent pain is required more often after single-fraction treatment (10-13). Results from a meta-analysis by Wu et al (10) including data from 8 randomized trials comparing single-fraction RT with 8-10 Gy to multifraction regimens with doses of 20-30 Gy delivered in 5-10 fractions showed similar response rates between groups. The overall and CR rates were $72.7 \%$ and $39.2 \%$ for patients treated with single-fraction RT and $72.5 \%$ and $40 \%$ for those treated with multifraction regimens. In the meta-analysis by Sze et al (11), which included 3508 patients from 12 randomized trials, overall response (OR) rates were $60 \%$ after single-fraction and $59 \%$ after multifraction RT and respective CR rates were $34 \%$ and $32 \%$. More recently, in a systematic review of 25 randomized trials comparing single-fraction versus different multifraction regimens, Chow et al (13) found similar response rates between treatment arms. Overall response rates were $72 \%$ in patients treated with single-fraction RT and $74 \%$ in those receiving multifraction regimens. Respective $\mathrm{CR}$ rates were $28 \%$ and $30 \%$. Taken together, these results support the use of single-fraction RT as an alternative to fractionated treatment and, on this basis, a recent guideline by the American Society for Therapeutic Radiology and Oncology recommended single fractions for uncomplicated bone metastases, as patient convenience is optimized (17). However, the authors suggested that multifraction RT could also be considered, as patients are less likely to require retreatment. In clinical practice, many physicians prefer to prescribe multifraction regimens (18). However, a careful selection of patients should be made and singlefraction or short-course multifraction schedules should be considered preferable in patients with poor performance status and/or limited life expectancy. Nevertheless, when possible, patient preference should also be considered in treatment decisions. Shakespeare et al (19) used a decision board instrument based on the Dutch Bone Metastasis Study to determine which fractionation schedule patients would choose between single-fraction or multifraction RT. The authors reported that $85 \%$ of patients chose the multifraction schedule because of lower retreatment and fracture rates compared to single-fraction RT, while the reasons for choosing the single-fraction treatment were cost or convenience. In contrast, Szumacher et al (20) found that $76 \%$ of patients preferred single-fraction RT because of convenience.
In the current study, we compared 2 multifraction RT schedules of $20 \mathrm{~Gy}$ in 5 fractions and $30 \mathrm{~Gy}$ in 10 fractions in patients with painful bone metastases. We found similar rates of pain relief between groups, suggesting that an abbreviated course of RT is as effective as long-course treatment for palliation of symptomatic bone metastases. The overall response rate at 1 month was $88.6 \%$. The overall response rate was $89.6 \%$ (CR and PR rates $6.5 \%$ and $83.1 \%$, respectively) in the $20-$ Gy arm compared with $87.3 \%$ (CR and PR rates $19 \%$ and $68.3 \%$, respectively) in the $30-$ Gy arm ( $p$ $=0.669$ ). The rate of CR was statistically better in patients treated with $30 \mathrm{~Gy}(p=0.019)$. The mean reduction in pain was 3.2 in the 20-Gy group and 3.6 in the $30-G y$ group. Pain progression was found in $6.5 \%$ of the irradiated lesions in the $20-$ Gy arm and in $1.6 \%$ in the $30-$ Gy arm. Our results compare favorably with previously reported series. Niewald et al (21) found no significant difference in pain relief in 100 patients randomly assigned to receive $20 \mathrm{~Gy}$ in 5 fractions or $30 \mathrm{~Gy}$ in 15 fractions. Complete response rates were $14 \%$ for the $20-$ Gy arm and $22 \%$ for the 30-Gy arm. However, despite a small trend favoring the 30-Gy treatment, the authors concluded that the 20-Gy schedule is preferable because of the limited life expectancy of patients with bone metastases. In a randomized trial of 150 patients treated with a single fraction of $8 \mathrm{~Gy}$ or multifractions of $20 \mathrm{~Gy}$ or $30 \mathrm{~Gy}$, El-Shenshawy et al (22) reported similar response rates among the 3 groups. The OR and CR rates were $78 \%$ and $32 \%$ in patients who received $20 \mathrm{~Gy}$ and $80 \%$ and $32 \%$ in those who received $30 \mathrm{~Gy}$, respectively. In a similar 3-arm randomized trial of 60 patients with bone metastases, Safwat et al (23) reported OR rates of $65 \%$ and $45 \%$ for patients treated with $20 \mathrm{~Gy}$ and $30 \mathrm{~Gy}$, respectively.

The main reason for using single-fraction or short-course RT in patients with bone metastases is that high radiation total doses may be associated with an increased risk of acute and late toxicity and this is of concern in patients with a worsened quality of life and a limited life expectancy. In our study, acute toxicity occurred in $2.6 \%$ of patients who received $20 \mathrm{~Gy}$ and in $23.8 \%$ of patients receiving $30 \mathrm{~Gy}$ $(p=0.001)$. In 272 patients randomly assigned to receive $8 \mathrm{~Gy}$ in single fraction or $20 \mathrm{~Gy}$ in 5 fractions, Roos et al (24) reported $2 \%$ of grade 3 toxicity in patients treated with 20 Gy. No grade 4 toxicity was observed. In another randomized trial, by Hartsell et al (25), comparing singlefraction (8 Gy) versus multifraction (30 Gy) RT in patients with breast or prostate cancer, grade 2-4 toxicities occurred in $17 \%$ of 433 patients who received $30 \mathrm{~Gy}$. In the randomized trial by El-Shenshawy et al (22), no significant difference in acute toxicity rate was observed between the 20-Gy and 30-Gy arm. More recently, in 160 patients randomly assigned to receive single-fraction RT of $8 \mathrm{~Gy}$ or multifraction treatment of $30 \mathrm{~Gy}$ in 10 fractions, Foro Arnalot et al (26) reported grade $1-2$ toxicity in $18 \%$ of patients treated with $30 \mathrm{~Gy}$. No grade 3-4 toxicity was observed.

The main long-term side effect of RT to the bone is the potential risk of pathologic fracture in the irradiated region. However, as suggested by Rades (27), it may sometimes be difficult to distinguish between fractures due to progression/ recurrence of osteolytic bone metastases and radiation-induced fractures. In our series, one case of pathologic fracture was 
observed in the 30-Gy arm. In a randomized trial of 239 patients treated with $8 \mathrm{~Gy}$ in single fraction or $20 \mathrm{~Gy}$ in 4 fractions, Nielsen et al (28) observed pathologic fractures in $5 \%$ of patients who received 20 Gy. Roos et al (24) reported a pathologic fracture rate of $4 \%$ for the $20-G y$ arm. Similar results were reported by Hartsell et al (25). In another randomized trial of 376 patients treated with $8 \mathrm{~Gy}$ or $30 \mathrm{~Gy}, 11 \%$ of patients who received 30 Gy experienced pathologic fracture of the irradiated bone (29).

Although single-fraction or short-course RT offer an effective treatment for palliation of bone metastases, reirradiation is often required for pain recurrence and a second course of RT may be associated with an increased risk of toxicity. Niewald et al (21) reported a re-irradiation rate of $2 \%$ for both $20-$ Gy and $30-$ Gy arms. In the study by Nielsen et al (28), $12 \%$ of patients treated with 20 Gy required reirradiation. El-Shenshawy et al (22) reported re-irradiation rates of $10 \%$ in the $20-$ Gy group and $4 \%$ in the $30-$ Gy group. In the trial by Foro Arnalot et al (26), 2\% of patients receiving $30 \mathrm{~Gy}$ were re-irradiated. Higher retreatment rates of $24 \%-25 \%$ for patients treated with 20 Gy and $9 \%-15 \%$ for patients who received 30 Gy have been reported in few other studies (23-25). In the current study, the re-irradiation rates were equivalent in both groups, but not statistically significant.

In conclusion, with the limitation of a retrospective study, our findings indicate that both $20 \mathrm{~Gy}$ in 5 fractions or $30 \mathrm{~Gy}$ in 10 fractions provide similar favorable symptomatic palliation of bone metastases. Complete response rate was higher in the 30-Gy group. Nevertheless, more intense RT is associated with higher toxicity, suggesting that an abbreviated course of $20 \mathrm{~Gy}$ could be preferred with the aim to reduce the risk of treatment-related complications while maintaining similar pain control. More randomized trials comparing different fractionation schedules of RT and including assessment of quality of life and cost-effectiveness are warranted to better determine the overall impact of these regimens and to guide physicians in the choice of the best treatment option.

\section{Disclosures}

Financial support: None.

Conflict of interest: None.

\section{References}

1. Tubiana-Hulin M. Incidence, prevalence and distribution of bone metastases. Bone. 1991;12(Suppl 1):S9-S10.

2. Galasko CSB. Incidence and distribution of skeletal metastases. Clin Orthop Relat Res. 1986;(210):14-22.

3. Coleman RE. Clinical features of metastatic bone disease and risk of skeletal morbidity. Clin Cancer Res. 2006;12(20 Pt 2): 6243s-6249s.

4. Bates T. A review of local radiotherapy in the treatment of bone metastases and cord compression. Int J Radiat Oncol Biol Phys. 1992;23(1):217-221.

5. Maher EJ, Coia L, Duncan G, Lawton PA. Treatment strategies in advanced and metastatic cancer: differences in attitude between the USA, Canada and Europe. Int J Radiat Oncol Biol Phys. 1992;23(1):239-244.

6. Mercadante $S$. Malignant bone pain: pathophysiology and treatment. Pain. 1997;69(1-2):1-18.
7. Cai B, Nickman NA, Gaffney DK. The role of palliative external beam radiation therapy in boney metastases pain management. J Pain Palliat Care Pharmacother. 2013;27(1):28-34.

8. Dennis K, Makhani L, Zeng L, Lam H, Chow E. Single fraction conventional external beam radiation therapy for bone metastases: a systematic review of randomised controlled trials. Radiother Oncol. 2013;106(1):5-14.

9. Lutz $\mathrm{S}$. The role of radiation therapy in controlling painful bone metastases. Curr Pain Headache Rep. 2012;16(4):300-306.

10. Wu JS, Wong R, Johnston M, Bezjak A, Whelan T; Cancer Care Ontario Practice Guidelines Initiative Supportive Care Group. Meta-analysis of dose-fractionation radiotherapy trials for the palliation of painful bone metastases. Int J Radiat Oncol Biol Phys. 2003;55(3):594-605.

11. Sze WM, Shelley M, Held I, Mason M. Palliation of metastatic bone pain: single fraction versus multifraction radiotherapy: a systematic review of the randomised trials. Cochrane Database Syst Rev. 2004;2(2):CD004721.

12. Chow E, Harris K, Fan G, Tsao M, Sze WM. Palliative radiotherapy trials for bone metastases: a systematic review. J Clin Oncol. 2007;25(11):1423-1436.

13. Chow E, Zeng L, Salvo N, Dennis K, Tsao M, Lutz S. Update on the systematic review of palliative radiotherapy trials for bone metastases. Clin Oncol (R Coll Radiol). 2012;24(2):112-124.

14. Steenland $\mathrm{E}$, Leer JW, van Houwelingen $\mathrm{H}$, et al. The effect of a single fraction compared to multiple fractions on painful bone metastases: a global analysis of the Dutch Bone Metastasis Study [erratum 2000;53:167]. Radiother Oncol. 1999;52(2): 101-109.

15. Farrar JT, Young JP Jr, LaMoreaux L, Werth JL, Poole RM. Clinical importance of changes in chronic pain intensity measured on an 11-point numerical pain rating scale. Pain. 2001;94(2): 149-158.

16. Cox JD, Stetz J, Pajak TF. Toxicity criteria of the Radiation Therapy Oncology Group (RTOG) and the European Organization for Research and Treatment of Cancer (EORTC). Int J Radiat Oncol Biol Phys. 1995;31(5):1341-1346.

17. Lutz S, Berk L, Chang E, et al; American Society for Radiation Oncology (ASTRO). Palliative radiotherapy for bone metastases: an ASTRO evidence-based guideline. Int J Radiat Oncol Biol Phys. 2011;79(4):965-976.

18. Bradley NM, Husted J, Sey MS, et al. Review of patterns of practice and patients' preferences in the treatment of bone metastases with palliative radiotherapy. Support Care Cancer. 2007;15(4):373-385.

19. Shakespeare TP, Lu JJ, Back MF, Liang S, Mukherjee RK, Wynne CJ. Patient preference for radiotherapy fractionation schedule in the palliation of painful bone metastases. J Clin Oncol. 2003;21(11):2156-2162.

20. Szumacher E, Llewellyn-Thomas H, Franssen E, et al. Treatment of bone metastases with palliative radiotherapy: patients' treatment preferences. Int J Radiat Oncol Biol Phys. 2005;61(5): 1473-1481.

21. Niewald M, Tkocz HJ, Abel U, et al. Rapid course radiation therapy vs. more standard treatment: a randomized trial for bone metastases. Int J Radiat Oncol Biol Phys. 1996;36(5): 1085-1089.

22. El-Shenshawy $\mathrm{H}$, El-Essawy KA. The effect of a single fraction compared to multiple fractions radiotherapy on painful bone metastases with evaluation of computed tomography bone density in osteolytic bone metastases. Bull Alex Fac Med. 2006; 42:439.

23. Safwat E, El-Nahas $\mathrm{T}$, Metwally $\mathrm{H}$, Abdelmotgally $\mathrm{R}$, Kassem $\mathrm{N}$. Palliative fractionated radiotherapy for bone metastases clinical and biological assessment of single versus multiple fractions. J Egypt Natl Cancer Inst. 2007;19:21e27. 
24. Roos DE, Turner SL, O'Brien PC, et al; Trans-Tasman Radiation Oncology Group, TROG 96.05. Randomized trial of $8 \mathrm{~Gy}$ in 1 versus 20 Gy in 5 fractions of radiotherapy for neuropathic pain due to bone metastases (trans-tasman radiation oncology group, TROG 96.05). Radiother Oncol. 2005;75: $54 \mathrm{e} 63$.

25. Hartsell WF, Scott CB, Bruner DW, et al. Randomized trial of short- versus long-course radiotherapy for palliation of painful bone metastases. J Natl Cancer Inst. 2005;97:798e804.

26. Foro Arnalot P, Fontanals AV, Galceran JC, et al. Randomized clinical trial with two palliative radiotherapy regimens in painful bone metastases: $30 \mathrm{~Gy}$ in 10 fractions compared with 8 Gy in single fraction. Radiother Oncol. 2008;89: 150 e155.

27. Rades D. Dose-Fractionation Schedules for Radiotherapy of Bone Metastases. Breast Care (Basel). 2010;5(5):339-344.

28. Nielsen OS, Bentzen SM, Sandberg E, Gadeberg CC, Timothy AR. Randomized trial of single dose versus fractionated palliative radiotherapy of bone metastases. Radiother Oncol. 1998; 47(3):233-240.

29. Kaasa S, Brenne E, Lund JA, et al. Prospective randomised multicenter trial on single fraction radiotherapy ( $8 \mathrm{~Gy} \times 1$ ) versus multiple fractions ( $3 \mathrm{~Gy} \times 10$ ) in the treatment of painful bone metastases. Radiother Oncol. 2006;79(3):278-284. 